\title{
An Assessment of MEG Coherence Imaging in the Study of Temporal Lobe Epilepsy
}

Kost Elisevich, M.D., Ph.D. ${ }^{1}$, Neetu Shukla, M.S. ${ }^{2}$, John Moran, Ph.D. ${ }^{3}$, Brien Smith, M.D..$^{3,4}$, Lonni Schultz, Ph.D. ${ }^{5}$, Karen Mason, R.EEG/MEG T. ${ }^{5}$, Gregory L Barkley, M.D. ${ }^{3,4}$, Norman Tepley, Ph.D. ${ }^{3}$, Valentina Gumenyuk, Ph.D. ${ }^{3}$, and Susan M. Bowyer, Ph.D. ${ }^{2,3,4}$

${ }^{1}$ Department of Neurosurgery, Henry Ford Health System, Detroit, Michigan, 48202 U.S.A.

2 Department of Physics, Oakland University, Rochester, Michigan, 48309 U.S.A.

${ }^{3}$ Department of Neurology, Henry Ford Health System, Detroit, Michigan, 48202 U.S.A.

4 Department of Neurology, Wayne State University, Detroit, Michigan, 48201 U.S.A.

${ }^{5}$ Department of Biostatistics and Research Epidemiology, Henry Ford Health System, Detroit, Michigan, 48202 U.S.A.

\section{Abstract}

Purpose-This study examines whether magnetoencephalographic (MEG) coherence imaging is more sensitive than the standard single equivalent dipole (ECD) model in lateralizing the site of epileptogenicity in patients with drug resistant temporal lobe epilepsy (TLE).

Methods-An archival review of ECD MEG analyses of 30 presurgical TLE patients was undertaken with data extracted subsequently for coherence analysis by a blinded reviewer for comparison of accuracy of lateralization. Postoperative outcome was assessed by Engel classification. MEG coherence images were generated from 10 minutes of spontaneous brain activity and compared to surgically resected brain areas outlined on each subject's MRI. Coherence values were averaged independently for each hemisphere to ascertain the laterality of the epileptic network. Reliability between runs was established by calculating the correlation between epochs. Match rates compared the results of each of the two MEG analyses with optimal postoperative outcome.

Key Findings-The ECD method provided an overall match rate of $43 \%$ (13 cases) for Engel class I outcomes with 37\% (11 cases) found to be indeterminate (i.e., no spikes identified on MEG). Coherence analysis provided an overall match rate of $77 \%$ (23 cases). Of 19 cases without evidence of mesial temporal sclerosis, coherence analysis correctly lateralized the side of TLE in 11 cases (58\%). Sensitivity of the ECD method was $41 \%$ (indeterminate cases included) and that of the coherence method $73 \%$ with a positive predictive value of $70 \%$ for an Engel class Ia outcome. Intrasubject coherence imaging reliability was consistent from run-to-run (correlation $>0.90$ ) using three 10-minute epochs.

Significance-MEG coherence analysis has greater sensitivity than the ECD method for lateralizing TLE and demonstrates reliable stability from run-to-run. It therefore improves upon the capability of MEG in providing further information of use in clinical decision-making where the laterality of TLE is questioned.

To the Editor: please use the address below concerning this MS. Susan Bowyer, Henry Ford Hospital, Neuromagnetism Lab CFP 78, 2799 West Grand Boulevard, Detroit, MI 48202, Tel. 313-916-1075, Fax. 313-916-0526, sbowyer1@ @hfhs.org.

Disclosures: None of the authors has any conflict of interest to disclose. We confirm that we have read the Journal's position on issues involved in ethical publication and affirm that this report is consistent with those guidelines. 


\section{Keywords}

Magnetoencephalography; interictal activity; single equivalent current dipole; presurgical assessment; neuronal network

\section{Introduction}

Magnetoencephalography (MEG) has gained acceptance as a noninvasive method for the study of focal epileptogenicity, particularly for those patients who are judged to have a drug resistant condition and for whom a surgical option exists (Barkley and Baumgartner, 2003; Burneo, 2004; Papanicolaou et al., 2004; Fischer et al., 2005; Shiraishi et al., 2005; Knowlton et al., 2006).

For temporal lobe epilepsy (TLE), a number of distinct electrographic (Williamson, 1987; Cascino et al., 1996; Cendes et al., 2000; Kilpatrick et al., 2003; Risinger et al., 1989; Lieb et al., 1976) and imaging (Bernasconi et al., 2000; Cendes et al, 1993; Jack et al., 1990; 1996; van Paesschen, 1997; Jafari-Khouzani et al., 2010) characteristics have been identified that provide ample but, at times, incomplete information for determining the laterality of the focus. Hence, further intracranial electrographic recording of both temporal lobes is often recommended. Even this application remains problematic because of surgical risk and sampling bias; moreover, the associated economic burden may be considerable (Elisevich et al., 1996). Whereas MEG has been most useful in detecting epileptiform activity over lateral neocortical areas (Knowlton \& Shih, 2004; Park et al., 2004; Bast et al., 2005), its application in cases of suspected mesial (m)TLE, the predominant site of surgical intervention for localization-related epilepsy, has been considered less so (Pataraia et al., 2005). The MR-FOCUSS source imaging technique (Moran et al. 2005) used in this study has a moderate depth imaging bias for cortical sources thus mitigating some of this concern. The effect of depth on coherence imaging was further reduced because the coherence calculation was normalized to remove the amplitude of sources. Single equivalent current dipole (ECD) modeling of interictal spike activity is commonly used for the estimation of generator sources (Bowyer et al., 2003; Ebersole \& Hawes-Ebersole, 2007) with the estimated ECDs superimposed on coregistered magnetic resonance (MR) images. The application is entirely dependent upon the recording of interictal discharges during the time of recording.

The detection of local and widespread epileptic network interactions may provide greater promise for determining the laterality of ictal onset zones and for defining the surgical target (Lehnertz et al., 2007). This is particularly useful in TLE cases in which there may be sufficient ambiguity in the electrographic and imaging analysis of the condition to confound the selection of the epileptogenic side. Epileptic network behavior has been shown to be associated with alterations in both gray and white matter in TLE (Bernasconi et al., 2004). However, measurements of physical connectivity (i.e., fiber tracts) and network signal (i.e., functional) connectivity have not yet been integrated and implemented in the framework of a complete brain imaging software package that is suitable for identifying a site for surgical resection.

Abnormal transients and oscillations of spontaneous neural activity can be found in MEG and EEG recordings. These transient waveforms and oscillations can be quantified by applying a time-frequency decomposition technique such as the short-time Fourier transform (stFFT) (Portnoff, 1980). After transformation to a time-frequency representation, the strength of network interactions can be estimated by the calculation of coherence which is a measure of synchrony between signals from different regions for each FFT frequency 
component (Nolte et al. 2004). The mean phase coherence (MPC), closely related to coherence, makes use of a Hilbert transform to extract the phase relationship between signals (Mormann et al., 2000). By calculating MPC between intracranial electrodes, Schevon et al. (2007) found regions of high phase coherence primarily located in the cerebral cortex adjacent to seizure onset zones. A seizure-free outcome was associated with removal of these highly coherent nodes (Schevon et al., 2007). Local increase of coherence during the interictal period has also been reported in particular regions of the temporal lobe (Towle et al., 1998; 1999). Increased levels of several synchronization measures in the involved hemisphere during the interictal period have also been reported (Kraskov, 2004). The finding that ictally connected neural networks can be detected directly from real-time MEG data raises the possibility of its use in the clinical investigation of localization-related epilepsy, in particular, its laterality in cases of TLE with confounding investigational features.

We undertook to study the utility of coherence analysis with MEG in confirming the laterality of the ictal onset zone in cases of TLE, a large proportion of which required inpatient extraoperative electrocorticography $(\mathrm{ECoG})$. A post hoc coherence analysis of each case by a blinded reviewer allowed for a comparison to be made with results obtained by conventional ECD analysis. We hypothesized that spontaneous recorded signals of an epileptic focus would be characterized by greater average coherence of local cortical activity because of its involvement in an underlying epileptic network. Accordingly, coherence analysis would provide greater sensitivity in lateralizing the TLE focus than the standard ECD methodology. Postoperative status provides a final determination of the accuracy in identifying laterality. Comparison of outcomes between the two methods of MEG analysis against that of an Engel class Ia status was used to assess the sensitivity of each application. A class Ia status comprises the most robust indicator of laterality (i.e., true positive) clinically and serves to distinguish which method best approximates this ideal. Ultimately, a prospective study will provide an overall measure of sensitivity by including all postoperative outcomes.

\section{Design and Methods}

\section{Patients}

An archival review of 30 patients (11 males, 19 females) with drug resistant TLE and ages at the time of MEG ranging from 8 to 59 years (mean $29 \pm 12$ years) was used in this study. The patients were selected consecutively from a listing of MEG studies performed over a five year period (2000 - 2005). A natural bias arose in this cohort as only those patients with conflicting or problematic investigational results (i.e., absence of mesial temporal sclerosis (MTS), artifact-related obscuration of ictal onset, differences in lateralization of epileptogenicity between EEG and imaging studies) were referred for MEG study. Standard investigation included inpatient video-electroencephalography (vEEG), MR imaging, single photon emission computed tomography (SPECT) ictal and interictal study, positron emission tomography (PET), sodium amobarbital study and a neuropsychological assessment. The criteria used for establishing MTS on MR imaging were as follows: (1) reduction of hippocampal volume as determined by a right-left asymmetry on T1-weighted coronal MR images, (2) increased FLAIR MR signal intensity and (3) loss of intrinsic hippocampal laminar structure. These features were required on at least two sequential coronal images to declare the impression of MTS. A single feature such as volume loss would therefore not support this impression. Further extraoperative electrocorticography (eECoG) with intracranial electrodes was performed in $17(57 \%)$ patients. Age at seizure onset ranged from infancy to 55 years (median 9.5 years; mean $12 \pm 12$ years) and the duration of epilepsy from 1 to 49 years (median 16.5 years, mean $18 \pm 12$ years). Table 1 summarizes the main clinical and imaging features of all patients. Postoperative outcome 
was assessed after one year using the Engel classification (Engel et al., 1982) by KE, GB and BJS. Twenty-two of the 30 patients (73\%) were identified as having a class Ia outcome. Of the remaining eight patients, four were subgrouped in class Ib-d and four in classes II and III. Eleven patients (37\%) were identified with MTS of whom ten had class Ia outcomes and one (case 2) had a class Id outcome.

This protocol was approved by the Institutional Review Board of Henry Ford Hospital.

\section{Control Subjects}

Eleven subjects ( 3 males, 8 females), aged 19 to 49 years (mean $30 \pm 10$ years), were screened by questionnaire and designated to be without neurological disorders. Age and gender distributions mirrored that of the study population. Control data were collected between 2007 and 2009. All were studied using the same MEG hardware and software as the study population.

\section{Magnetoencephalography Data Analysis}

As part of a routine presurgical evaluation, three 10 minute epochs of MEG and EEG data were acquired from each patient at a sample rate of $508.6 \mathrm{~Hz}$ DC to $100 \mathrm{~Hz}$ analog filtered using a 148 channel whole head magnetometer (4D Neuroimaging, San Diego CA) in a magnetically shielded room. Control subjects had 10 minute MEG recordings performed in the same manner. During these 10 minutes, spontaneous brain activity was collected while the subject was in a resting state (i.e., awake, drowsy and/or sleep activity).

The original analysis of data included a filtering at 3-50 Hz, performance of a single ECD analysis and display of the results on MR images which were coregistered to the subject's digitized head shape recorded at the time of MEG data collection. Automatic probe position routines were conducted to locate the head with respect to the neuromagnetometer detector coils. The whole head helmet containing the detector array was placed in close proximity to most of the cerebrocortical surface. Changes in the subject's position during the study were detected by alterations in magnetic field locations from coils on the forehead and ears. Runs during which the subject moved more than $0.8 \mathrm{~cm}$ were repeated (Bowyer et al., 2003).

Magnetoencephalographic data for control subjects were collected and filtered similarly at 3-50 Hz. Single ECD analysis was not performed on MEG data from control subjects as no spikes were identified.

The frequency dependency of coherence conveys information only about the natural frequency characteristics of the corresponding brain network as the imaged pattern of coherence across the brain is relatively unaltered across frequencies. Across all patients, the spatial distribution of coherence between frequencies correlated greater than 0.98 . This justified averaging coherence across frequencies since the average contained all the information about the distribution of coherence in the brain. The average coherence is different from a correlation analysis of brain signals as coherence eliminates the problem of time lags of activation between brain regions. The frequency range with the greatest power tended towards the lower end of band-passed data, corresponding to alpha activity in the 9 to $14 \mathrm{~Hz}$ frequency band. There was only a weak relationship between power spectral amplitude and coherence especially for locations characterized by high coherence.

\section{Single Equivalent Current Dipole Analysis}

Magnetoencephalographic parameters used to establish whether the ECD application was reliable included the following selection criteria: (1) correlation coefficient (R) greater than 0.98 [degree of association between the actual measured magnetic field data and the estimated forward solution data]; (2) goodness-of-fit (GoF) greater than 0.95 [measure of 
how well the defined magnetic field agrees with that estimated from an assumed dipole]; (3) root mean square (RMS) greater than 400fT [measure of magnetic signal strength of the data at the latency for which the dipole is computed]; (4) dipole moment (Q) greater than 200nAm [strength of the dipole vector]; and, (5) confidence volume (CV) less than $1.0 \mathrm{~cm}^{3}$ [spatial volume encompassing the $95 \%$ probability of source localization (Bowyer et al. 2003).

\section{Coherence Analysis}

For the current post hoc coherence analysis, the digitally filtered MEG data (band pass 3-50 $\mathrm{Hz}$ ) for both patients and control subjects were reloaded into the MEG Tools software (Moran, 2008). The band pass filter eliminated the very low frequency background variations and reduced the high frequency environmental magnetic noise. A fourth order ICA artifact filter was used to remove heart signals observed in the MEG recordings.

To localize cortical source activation of epileptiform activity, a model of gray matter was constructed using each individual's T1-weighted high-resolution volumetric MR image. The realistic head model consisted of X-, Y- and Z- oriented dipoles at approximately 4000 locations distributed to represent the same amount of gray matter identified in each individual's MR image. These MR images were coregistered with the individual's digitized head shape recorded at the time of MEG data collection.

The 10 minutes of rest state MEG data were prepared for source imaging by division into 80 segments, each containing 7.5 seconds of data of relatively uniform brain behavior (Moran et al., 2004; 2005). For each of these data segments, signals from neuronal sources were isolated using an ICA spatiotemporal decomposition technique designed to extract signals from distinct compact sources that exhibit burst behavior and minimal temporal overlap with other active sources. These ICA signal components have MEG spatial magnetic field patterns corresponding to one or a few spatially distinct compact sources which are much easier to image accurately using the MR-FOCUSS source imaging technique (Moran et al., 2005). Separate from the imaging algorithm, the cross-spectrum between ICA signals was calculated. In these cross-spectrum calculations, a sequence of FFT spectra was calculated using $0.5 \mathrm{sec}$ windows and 25\% overlap with FFT amplitudes for 24 frequency bins of $2 \mathrm{~Hz}$ width between 3 and $50 \mathrm{~Hz}$. The imaging results and the signal cross-spectrum were used to calculate the coherence between all pairings of active cortical locations within each of the 24 frequency bins. Finally, for each active source, the average coherence across frequencies and sources was calculated. In these coherence imaging results, the localization of imaged brain activity is strongly dependent on the frequency bands with greatest power.

A detailed coherence calculation is included in the Appendix (online). Laterality was determined by calculating the mean coherence values for each individual hemisphere. The coherence value of each source in a hemisphere was summed and divided by the number of sources.

\section{Identification of High MEG Coherence}

High coherence was expressed by red color coding (Fig. 1, 2). Individual scaling was necessary to demonstrate locations of activity relative to each figure's maximum amplitude. A fixed scale would compromise the ability to determine the scale invariant distribution of source amplitude conveyed in a figure for any individual. The resection volume was schematically outlined on the preoperative MR image of each patient by the neurosurgeon (KE). Images were inspected by a senior epileptologist (BJS) blinded to patient identity and postoperative outcome. The location of maximal MEG coherence was identified as 
unilateral temporal, bilateral temporal or bilateral extratemporal (Table 2). High coherence (i.e., red) areas that matched with the surgical resection site were marked ' $Y$ '.

\section{Statistical Analysis}

The match rates for the ECD and the coherence imaging methods were compared using McNemar's test (Fleiss et al., 1986). Only those patients with class I outcomes were used in the comparison. A two-sample t-test was performed to compare the means from coherence analysis between control subjects and patients with TLE. A similar comparison of means established between the operated and contralateral hemispheres was assessed using the paired t-test. To assess the consistency of coherence imaging across runs, correlation coefficients were computed.

\section{Results}

\section{Magnetic Resonance Images and Surgical Outcomes}

Class I outcomes were identified in 26 cases (87\%) out of the group of 30 patients (Table 2). Class Ia outcomes comprised the great majority of these $(22 / 26,85 \%)$. Four patients retained disabling seizure activity postoperatively rendering suboptimal Engel classifications [cases 7 (IIb), 10 (IIIa), 14 (IIIa), 21 (IIa)]. Of the 19 cases in which MTS was not apparent on MR imaging, coherence analysis provided localizing data that identified a class I outcome in 11 $(58 \%, 11 / 19)$. In the remaining eight cases $(42 \%)$, coherence analysis was not useful in five (cases 3, 11, 14, 29, 30) and identified incorrectly the site of epileptogenicity in three (cases $7,10,21)$.

\section{Single Equivalent Current Dipole Analysis}

No epileptiform activity was identified in 11 patients (37\%) and, of the 19 remaining cases with ECD localizations, $13(68 \%, 13 / 19)$ achieved class I outcomes (Table 2). Of the entire study population of 30 patients, ECD analysis provided information indicating a favorable outcome (i.e., class I) in 50\%. Sixteen cases matched, by laterality, with the side of the resection although not all achieved favorable outcomes (Table 3). Nine patients had a class Ia outcome with an additional four patients achieving class Ib-d outcomes and three others, class II or III outcomes. Of the 14 patients who could not be localized, 11 had no epileptiform activity but achieved class Ia outcomes, one (case 3) showed dipoles contralateral to the side of resection (i.e., left temporal) in the right frontal lobe in addition to the left insula and two (cases 21,25) lateralized correctly but with insufficient clustering of dipoles in the temporal lobe. Despite this lack of concordance, two of the three latter patients (cases 3,25) achieved a class Ia outcome whereas the remaining patient (case 21) had a class IIa outcome.

\section{Coherence Analysis}

The pattern of coherence across the cortical structure was nearly identical for frequencies from 3 to $50 \mathrm{~Hz}$ in each subject. The mean coherence across all 24 frequency bins, within the $3-50 \mathrm{~Hz}$ band, was calculated and sites of coherence coregistered on the presurgical MR images (Figs 1,2). These sites coincided with the respective temporal lobe undergoing resection in $23(77 \%)$ of the 30 patients examined (Table 2). An interhemispheric comparison of means from coherence analysis of all 30 patients identified a value of 0.089 $( \pm 0.013)$ for the resected side and $0.074( \pm 0.011)$ for the contralateral side. The difference in means between the two sides was statistically significant ( $\mathrm{p}<0.001$; Fig 3$)$. The results identify agreement with those of the prior conventional investigation although do not correlate with optimal postoperative outcome in all cases. Using only the class I outcome results (i.e., absence of disabling seizures) as a measure of success, the mean coherence 
value was found to be $0.26 \pm 0.03$. Control subjects demonstrated no area of high coherence, as expected, and a lower mean coherence value $(0.17 \pm 0.09)$. A diffuse pattern appeared scattered over and near the cerebral mantle in control subjects (Supplemental Fig. 1; available online). This difference in means between the two groups was statistically significant $(\mathrm{p}=0.007)$ (Supplemental Fig. 2; available online).

Coherence analysis lateralized the site of temporal epileptogenicity in 16 (73\%) of 22 TLE patients who achieved a class Ia outcome following surgery (Table 3). Of the 11 patients identified without epileptiform activity by the ECD method, eight could still be analyzed and localized effectively using coherence analysis to achieve a class Ia outcome (Fig. 2, Table 2). Of the seven patients (cases 3,11, 12, 14, 16, 29, 30) who could not be lateralized by coherence analysis, three (cases $11,12,29$ ) showed no epileptiform activity during the study and could not be analyzed by the ECD method, whereas, three others (cases 14, 16, 30) were correctly lateralized. In the final patient (case 3), neither method was sufficient. Despite such discrepancies, all patients achieved class Ia outcomes except case 14 (IIIa). A detailed examination of the nature of the findings of coherence analysis indicated, that in four patients (cases 11, 16, 29, 30), a pattern of near symmetric bitemporal coherence was obtained with greater prominence contralateral to the side of resection. Two patients (cases 3,14 ) showed symmetric bitemporal coherence without any indication of lateralization. In the final patient (case 12), lateralization was correct for the right hemisphere but without sufficient concentration of signal in the temporal lobe to declare it specifically epileptogenic. Assessment of the 26 patients with class I outcomes showed a match rate for the coherence method of $76.9 \%$ compared with a $50 \%$ match rate for the ECD method $(\mathrm{p}=0.065)$.

\section{Sensitivity and Specificity}

The sensitivity and specificity were determined for each of the two methods of MEG analysis using Engel class Ia outcomes as an absolute measure of success (Table 4). In the case of the ECD method, 11 cases were indeterminate for lack of epileptiform activity and their inclusion necessarily underestimates the sensitivity of the method as it raises the false negative prediction from two (cases 3,25 ) to 13 cases and lowers the sensitivity from $82 \%$ $(9 / 11)$ in the case of their exclusion to $41 \%$ (9/22) with their inclusion. A specificity of $13 \%$ (1/8) was calculated for the ECD method. Using coherence analysis, there were no indeterminate predictions and six false negative results. With 16 true positive results identified, a sensitivity of $73 \%$ was calculated. A specificity of $13 \%(1 / 8)$ was found with seven false positive results identified. Coherence analysis provided a positive predictive value for Engel class Ia of 70\% (16/23), whereas for ECD, it was 56\% (9/16).

\section{Coherence Reliability}

A reliability study was performed to establish whether coherence imaging measurements were consistent from run-to-run. For each subject, multiple runs of three separate ten minute epochs were collected with an interval of five minutes between epochs. Correlation of coherence from run-to-run was found to be greater than 0.90 for all subjects (Supplemental Fig. 3; available online). Little difference in coherence was detected for runs performed during awake and drowsy states with a correlation of 0.92 .

\section{Discussion}

Coherence analysis using MEG provides superior lateralization of a site of epileptogenicity in cases of TLE when compared to the more commonly used ECD method. The analysis is not limited by an absence of epileptiform activity during the course of the study and, because of its ability to identify higher synchronization of activity in the seizure onset zone (Arnhold et al., 1999; Mormann et al., 2000; Bartolomei et al., 2004), the method is 
applicable in all patients undergoing investigation for partial epilepsy. In fact, one third of patients in this study were excluded from analysis by the ECD method for lack of epileptiform activity. Identification of class I outcomes using either method was similar when these indeterminate results were excluded from analysis in the case of the ECD method. When included, the ability of the ECD method to identify a class I outcome fell from $87 \%$ to $50 \%$. Similarly, because of the indeterminacy problem, the sensitivity of the ECD methodology was found to be $41 \%$ as compared to $73 \%$ with coherence analysis. The specificities for each method are low indicating that neither provides sufficient exclusionary information in assessing the laterality of focal temporal epileptogenicity.

Although coherence analysis correctly identified an Engel class I outcome in 20 (67\%) of 30 cases, it incorrectly identified a putative site of epileptogenicity corresponding to that determined by standard investigation in three cases $(7,10$, and 21$)$ that had achieved suboptimal results (i.e., Engel class II, III) assuming that a sufficient resection had been performed. Furthermore, it provided misleading information regarding the laterality of epileptogenicity in another six cases that achieved optimal results (i.e., Engel class Ia). In a large retrospective review of 455 patients with partial epilepsy of both temporal and extratemporal origin, an average sensitivity of MEG for specific epileptic activity, using the ECD method was $76.3 \%$ with the exclusion of indeterminate cases (Stefan et al., 2003). This was not, however, a final confirmation of epileptic focality amenable to surgical resection and, therefore, was not fully verifiable in accordance with surgical outcome. Of 109 patients with TLE who did undergo resection in the latter study, ECD analysis identified the corresponding lobe in 94 (86.2\%) cases. Engel class I and II categories were grouped together and regarded as a successful outcome and no numerical distribution between the categories was provided. A similar grouping of class I and II outcomes in the current study yields six more cases, four with class Ib-d and two with class II outcomes, for a total of 28 cases. Of this total, ECD analysis achieved a match rate of $64 \%$ and coherence analysis, 79\%. Apart from the disparity in study populations (i.e., 28 vs 109 cases), the relative distributions of class I and II outcomes and the manner of case selection may account for differences between the two studies.

A limitation of this MEG study was the lack of information regarding coherence variability during different states of arousal (i.e., wakeful, drowsy, asleep) and the lack of standardization of measures in these states. Interictal spike discharges are more common during drowsiness and light sleep. One would expect the yield of ECD MEG analysis and perhaps the magnitude of coherence to be greater in the latter condition. Intersubject variability in arousal during recording may have affected ECD measures adversely to bring about lower sensitivity in this study. Run-to-run variability, however, did not seem to bear this out. Moreover, coherence analysis remained the application with the greater yield for the same interval of recording. Postictal recording with its greater likelihood of spike discharge may be a further consideration in this argument that would ostensibly improve yield with both analyses (Oishi et al., 2002).

From a clinical perspective, the ability to salvage data in the absence of concurrent epileptiform activity bears considerable practical importance; however, its place in the investigation of TLE must be examined alongside other applications serving to do the same. Innovative postprocessing of MR imaging applications and electrographic approaches have been developed in order to better establish the laterality of TLE preoperatively. Volumetric assessment of the medial temporal structures (Jack et al., 1990; Cendes et al., 1993; Kim et al., 1994) has been used, for some time, as a quantitative measure to establish the laterality of an epileptic focus as volume reduction of the hippocampus, in particular, has been shown to coincide with the location of the focus. However, in $15-30 \%$ of mTLE cases, no distinct asymmetry in volume can be established (Jackson et al., 1994; Paesschen, 1997; Carne et al., 
2004). An increase in signal intensity in both T2-weighted and fluid-attenuated inversion recovery (FLAIR) images (Jack et al., 1996) has also provided lateralizing information and a recent study of mean and standard deviation of FLAIR signal intensity (Jafari-Khouzani et al., 2010) has indicated accurate lateralization in $98 \%$ of proven mTLE cases. There is evidence, however, of cases in which MTS was identified by MR imaging but no mTLE was found. This has been specifically noted in familial mTLE in which upwards of $34 \%$ of asymptomatic first degree relatives of patients with mTLE were found to have MTS (Kobayashi et al., 2003). The application of texture analysis in MR imaging to mTLE has also shown promise over that of volumetry (Yu et al., 2001; Bonilha et al., 2003; JafariKhouzani et al., 2010) The apparent diffusion coefficient (ADC) is significantly reduced in mTLE with diffusion-weighted MR imaging (O'Brien et al., 2007); however, in certain cases without distinct lateralizing features identified by conventional MR imaging, no difference in ADC has been identified between the temporal lobes (Wehner et al., 2007). Other imaging modalities, particularly functional studies, have therefore been used in a complementary fashion to arrive at a more accurate predictive standard in the case of TLE. Subtraction ictal SPECT coregistered to MRI (SISCOM) may be of use in localizing epileptic foci (Spanaki et al., 1999), sometimes obviating the need for intracranial electrode placement (Tan, 2008). Positron emission tomography (PET) of serotonin $\left(\mathrm{HT}_{1 \mathrm{~A}}\right)$ receptor uptake binding may be reduced in TLE (Didelot et al., 2008) even in the absence of distinct pathological change (Merlet et al., 2004). ECD source localization has been adopted in a similar capacity with favorable postoperative outcomes reported when the resection volume included or was proximate to ellipsoidal volumes containing a cluster of dipoles (Fischer et al., 2005).

The use of MEG in the investigation of TLE does offer unique information toward the lateralization of the epileptic focus in cases where ambiguity exists in determining which of the two temporal lobes is epileptogenic after completion of standard study. This appears to be particularly the case with the application of coherence analysis as was seen with significant interhemispheric differences in coherence in the present study. A match rate of $76.9 \%$ was achieved for class I outcomes. However, in TLE cases without MTS, of which there were 19 in this study, a match rate of only $58 \%$ was achieved. With coherence analysis identified as the better predictor of laterality in TLE, its overall sensitivity may be established in a prospective study that includes all clinical outcomes. Additionally, further insight might be obtained in specific cases where coherence analysis fails to adequately achieve acceptable lateralization and disagrees with the accrued data from standard investigation that has suggested otherwise (i.e., case 14). Two cases were noted in our study population to have had particularly poor outcomes (i.e., class IIIa). Each had widespread hemispheric changes on MR imaging. In case 10, coherence analysis identified network activity more distributed in the frontotemporal region and in case 14 , symmetric bitemporal coherence was apparent. Such cases may indeed deserve closer scrutiny in order to avoid suboptimal postoperative outcomes.

At the present time, there is no single investigational modality that can reliably identify the location of an epileptic focus in those cases of TLE in which there appears to be ambiguity regarding the side of ictal onset. In fact, each modality, including MEG, may provide misleading information that improperly lateralizes the focus. With continuing improvements in these individual applications, however, and their proper use in a complementary fashion, more dependable lateralization of temporal lobe epileptogenicity in the more challenging cases may be possible. The application of MEG coherence analysis offers advantages over that of the ECD method but still falls short of providing the needed predictive strength for concluding laterality. Progress along these lines, however, may ultimately arrive at an investigational model sufficiently robust to circumvent the need for extraoperative ECoG in the majority of patients. 


\section{Supplementary Material}

Refer to Web version on PubMed Central for supplementary material.

\section{Acknowledgments}

This research was supported by NIH/NINDS Grant R01 NS30914 (Tepley).

\section{References}

Arnhold J, Grassberger P, Lehnertz K, Elger CE. A robust method for detecting interdependences: application to intracranially recorded EEG. Phys D. 1999; 134:419-430.

Barkley GL, Baumgartner C. MEG and EEG in epilepsy. J Clin Neurophysiol. 2003; 20:163-178. [PubMed: 12881663]

Bartolomei F, Wendling F, Regis J, Gavaret M, Guye M, Chauvel P. Pre-ictal synchronicity in limbic networks of mesial temporal lobe epilepsy. Epilepsy Res. 2004; 61:89-104. [PubMed: 15451011]

Bast T, Ramantani G, Boppel T, Metzke T, Ozkan O, Stippich C, Seitz A, Rupp A, Rating D, Scherg M. Source analysis of interictal spikes in polymicrogyria: loss of relevant cortical fissures requires simultaneous EEG to avoid MEG misinterpretation. NeuroImage. 2005; 25:1232-1241. [PubMed: 15850741]

Bernasconi A, Bernasconi N, Caramanos Z, Reutens DC, Andermann F, Dubeau F, Tampieri D, Pike BG, Arnold DL. T2 relaxometry can lateralize mesial temporal lobe epilepsy in patients with normal MRI. NeuroImage. 2000; 12:739-746. [PubMed: 11112405]

Bernasconi N, Duchesne S, Janke A, Lerch J, Collins DL, Bernasconi A. Whole-brain voxel-based statistical analysis of gray matter and white matter in temporal lobe epilepsy. NeuroImage. 2004; 23:717-723. [PubMed: 15488421]

Bonilha L, Kobayashi E, Castellano G, Coelho G, Tinois E, Cendes F, Li M. Texture analysis of hippocampal sclerosis. Epilepsia. 2003; 44:1546-1550. [PubMed: 14636326]

Bowyer SM, Mason K, Tepley N, Smith B, Barkley GL. Magnetoencephalographic validation parameters for clinical evaluation of interictal epileptic activity. J Clin Neurophysiol. 2003; 20:8793. [PubMed: 12766680]

Burneo JG, Bebin M, Kuzniecky RI, Knowlton RC. Electroclinical and magnetoencephalographic studies in epilepsy patients with polymicrogyria. Epilepsy Res. 2004; 62:125-133. [PubMed: 15579301]

Carne RP, O'Brien TJ, Kilpatrick CJ, MacGregor LR, Hicks RJ, Murphy MA, Bowden SC, Kaye AH, Cook MJ. MRI-negative PET-positive temporal lobe epilepsy: a distinct surgically remediable syndrome. Brain. 2004; 127:2276-2285. [PubMed: 15282217]

Cascino GD, Trenerry MR, So EL, Sharbrough FW, Shin C, Lagerlund TD, Zupanc ML, Jack CR. Routine EEG and temporal lobe epilepsy: relation to long-term EEG monitoring quantitative MRI and operative outcome. Epilepsia. 1996; 37:651-656. [PubMed: 8681897]

Cendes F, Leproux F, Melanson D, Ethier R, Evans A, Peters T, Andermann F. MRI of amygdala and hippocampus in temporal lobe epilepsy. J Comput Assist Tomogr. 1993; 17:206-210. [PubMed: 8454746]

Cendes F, Li LM, Watson C, Andermann F, Dubeau F, Arnold DL. Is ictal recording mandatory in temporal lobe epilepsy? Not when the interictal electroencephalogram and hippocampal atrophy coincide. Arch Neurol. 2000; 57:497-500. [PubMed: 10768623]

de Pasquale F, Della Penna S, Snyder AZ, Lewis C, Mantini D, Marzetti L, Belardinelli P, Ciancetta L, Pizzella V, Romani GL. Temporal dynamics of spontaneous MEG activity in brain networks. Proc Nat Acad Sci. 107:6040-6045.

Didelot A, Ryvlin P, Lothe A, Merlet I, Hammers A, Mauguiere F. PET imaging of brain 5-HT1A receptors in the preoperative evaluation of temporal lobe epilepsy. Brain. 2008; 131:2751-2764. [PubMed: 18790822]

Ebersole JS, Hawes-Ebersole S. Clinical application of dipole models in the localization of epileptiform activity. J Clin Neurophysiol. 2007; 24:120-129. [PubMed: 17414967] 
Elisevich K, Kasunic K, Hathaway SJ, Smith BJ, Stewart RW, Barkley GL. Clinical care pathways in a surgical epilepsy program: A comparison of costs by diagnostic related groups for program and nonprogram admissions. J Epilepsy. 1996; 9:282-290.

Engel JJ, Troupin AS, Crandall PH, Sterman MB, Wasterlain CG. Recent developments in the diagnosis and therapy of epilepsy. Ann Intern Med. 1982; 97:584-598. [PubMed: 6812475]

Fischer MJ, Scheler G, Stefan H. Utilization of magnetoencephalography results to obtain favourable outcomes in epilepsy surgery. Brain. 2005; 128:153-157. [PubMed: 15563514]

Fleiss, JL. The design and analysis of clinical experiments. New York: John Wiley \& Sons; 1986.

Jack CR Jr, Rydberg CH, Krecke KN, Trenerry MR, Parisi JE, Rydberg JN, Cascino GD, Riederer SJ. Mesial temporal sclerosis: diagnosis with fluid-attenuated inversion-recovery versus spin-echo MR imaging. Radiology. 1996; 199:367-373. [PubMed: 8668780]

Jack CR Jr, Sharbrough FW, Twomey CK, Cascino GD, Hirschorn KA, Marsh WR, Zinsmeister AR, Scheithauer B. Temporal lobe seizures: lateralization with MR volume measurements of the hippocampal formation. Radiology. 1990; 175:423-429. [PubMed: 2183282]

Jackson GD, Kuzniecky RI, Cascino GD. Hippocampal sclerosis without detectable hippocampal atrophy. Neurology. 1994; 44:42-46. [PubMed: 8290088]

Jafari-Khouzani K, Elisevich K, Patel S, Smith B, Soltanian-Zadeh H. FLAIR signal and texture analysis for lateralizing mesial temporal lobe epilepsy. Neuroimage. 2010; 49:1559-1571. [PubMed: 19744564]

Kilpatrick C, O'Brien T, Matkovic Z, Cook M, Kaye A. Preoperative evaluation of temporal lobe surgery. J Clin Neurosci. 2003; 10:535-539. [PubMed: 12948454]

Kim JH, Tien RD, Felsberg GJ, Osumi AK, Lee N. MR measurements of the hippocampus for lateralization of temporal lobe epilepsy: value of measurements of the body vs the whole structure. AJR Am J Roentgenol. 1994; 163:1453-1457. [PubMed: 7992746]

Knowlton RC, Elgavish R, Howell J, Blount J, Burneo JG, Faught E, Kankirawatana P, Riley K, Morawetz R, Worthington J, Kuzniecky R. Magnetic source imaging versus intracranial electroencephalogram in epilepsy surgery: a prospective study. Ann Neurol. 2006; 59:835-842. [PubMed: 16634031]

Knowlton RC, Shih J. Magnetoencephalography in epilepsy. Epilepsia. 2004; 45:61-71. [PubMed: 15281961]

Kobayashi E, D'Agostino MD, Lopes-Cendes I, Berkovic SF, Li ML, Andermann E, Andermann F, Cendes F. Hippocampal atrophy and T2-weighted signal changes in familial mesial temporal lobe epilepsy. Neurology. 2003; 60:405-409. [PubMed: 12578919]

Kraskov, A. PhD thesis. John Von Neumann Institute for Computing, NIC; 2004. Applications of synchronization and interdependence measures in particular to EEG of epilepsy patients.

Lieb JP, Walsh GO, Babb TL, Walter RD, Crandall PH. A comparison of EEG seizure patterns recorded with surface and depth electrodes in patients with temporal lobe epilepsy. Epilepsia. 1976; 17:137-160. [PubMed: 947745]

Lehnertz K, Mormann F, Osterhage H, Muller A, Prusseit J, Chernihovskyi A, Staniek M, Krug D, Bialonski S, Elger CE. State-of-the-art of seizure prediction. J Clin Neurophysiol. 2007; 24:147153. [PubMed: 17414970]

Merlet I, Ostrowsky K, Costes N, Ryvlin P, Isnard J, Faillenot I, Lavenne F, Dufournel D, Le Bars D, Mauguiere F. 5-HT1A receptor binding and intracerebral activity in temporal lobe epilepsy: an [18F]MPPF-PET study. Brain. 2004; 127:900-913. [PubMed: 14985263]

Moran, JE. Detroit: 2008. MEG Tools. www.megimaging.comMEG signal processing, data visualization, MRI integration, brain imaging and visualization

Moran JE, Bowyer S, Tepley N. Multi-Resolution FOCUSS: A source imaging technique applied to MEG data. Brain Topography. 2005; 18:1-17. [PubMed: 16193262]

Moran JE, Drake CL, Tepley N. ICA methods for MEG imaging. J Neurol Clin Neurophysiol. 2004; 72:1-6.

Mormann F, Lehnertz K, David PE, Elger C. Mean phase coherence as a measure for phase synchronization and its application to the EEG of epilepsy patients. Phys D. 2000; 144:358-369. 
Nolte G, Bai O, Wheaton L, Mari Z, Vorbach S, Hallett M. Identifying true brain interaction from EEG data using the imaginary part of coherency. Clin Neurophysiol. 2004; 115:2292-2307. [PubMed: 15351371]

O'Brien TJ, David EP, Kilpatrick CJ, Desmond P, Tress B. Contrast-enhanced perfusion and diffusion MRI accurately lateralize temporal lobe epilepsy: A pilot study. J Clin Neurosci. 2007; 14:841849. [PubMed: 17596947]

Oishi M, Kameyama S, Morota N, Tomikawa M, Wachi M, Kakita A, Takahashi H, Tanaka R. Fusiform gyrus epilepsy: the use of ictal magnetoencephalography. J Neurosurg. 2002; 97:200204. [PubMed: 12134913]

Paesschen WV. Quantitative MRI of mesial temporal structures in temporal lobe epilepsy. Epilepsia. 1997; 38:3-12.

Papanicolaou AC, Simos PG, Castillo EM, Breier JI, Sarkari S, Pataraia E, Billingsley RL, Buchanan $\mathrm{S}$, Wheless J, Maggio V, Maggio WW. Magnetocephalography: a noninvasive alternative to the Wada procedure. J Neurosurg. 2004; 100:867-876. [PubMed: 15137606]

Park HM, Nakasato N, Iwasaki M, Shamoto H, Tominaga T, Yoshimoto T. Comparison of magnetoencephalographic spikes with and without concurrent electroencephalographic spikes in extratemporal epilepsy. Tohoku J Exp Med. 2004; 203:165-174. [PubMed: 15240925]

Pataraia E, Lindinger G, Deecke L, Mayer D, Baumgartner C. Combined MEG/EEG analysis of the interictal spike complex in mesial temporal lobe epilepsy. NeuroImage. 2005; 24:607-614. [PubMed: 15652296]

Portnoff M. Time-frequency representation of digital signals and systems based on short-time fourier analysis. IEEE Trans Acoust Speech Signal Proces. 1980; 28:55-69.

Risinger MW, Engel J Jr, Van Ness PC, et al. Ictal localization of temporal lobe seizures with scalp/ sphenoidal recordings. Neurology. 1989; 39:1288-1293. [PubMed: 2797451]

Schevon CA, Cappell J, Emerson R, Isler J, Grieve P, Goodman R, McKhann G Jr, Weiner H, Doyle W, Kuzniecky R, Devinsky O, Gilliam F. Cortical abnormalities in epilepsy revealed by local EEG synchrony. NeuroImage. 2007; 35:140-148. [PubMed: 17224281]

Shiraishi H, Ahlfors SP, Stufflebeam SM, Takano K, Okajima M, Knake S, Hatanaka K, Kohsaka S, Saitoh S, Dale AM, Halgren E. Application of magnetoencephalography in epilepsy patients with widespread spike or slow-wave activity. Epilepsia. 2005; 46:1264-1272. [PubMed: 16060938]

Spanaki MV, Spencer SS, Corsi M, MacMullan J, Seibyl J, Zubal IG. Sensitivity and specificity of quantitative difference SPECT analysis in seizure localization. J Nucl Med. 1999; 40:730-736. [PubMed: 10319743]

Stefan H, Hummel C, Scheler G, Genow A, Druschky K, Tilz C, Kaltenhauser M, Hopfenartner R, Buchfelder M, Romstock J. Magnetic brain source imaging of focal epileptic activity: a synopsis of 455 cases. Brain. 2003; 126:2396-2405. [PubMed: 12876149]

Tan KM, Britton JW, Buchhalter JR, Worrell GA, Lagerlund TD, Shin C, Cascino GD, Meyer FB, So EL. Influence of subtraction ictal SPECT on surgical management in focal epilepsy of indeterminate localization: a prospective study. Epilepsy Res. 2008; 82:190-193. [PubMed: 18835758]

Towle VL, Carder RK, Khorasani L, Lindberg D. Electrocorticographic coherence patterns. J Clin Neurophysiol. 1999; 16:528-547. [PubMed: 10600021]

Towle VL, Syed I, Berger C, Grzesczcuk R, Milton J, Erickson RK, Cogen P, Berkson E, Spire JP. Identification of the sensory/motor area and pathologic regions using ECoG coherence. Electroencephalogr Clin Neurophysiol. 1998; 106:30-39. [PubMed: 9680162]

Van Paesschen W, Sisodiya S, Connelly A, Duncan JS, Free SL, Raymond AA, Grunewald RA, Revesz T, Shorvon SD, Fish DR, et al. Quantitative hippocampal MRI and intractable temporal lobe epilepsy. Neurology. 1995; 45:2233-2240. [PubMed: 8848199]

Wehner T, Lapresto E, Tkach J, Liu P, Bingaman W, Prayson RA, Ruggieri P, Diehl B. The value of interictal diffusion-weighted imaging in lateralizing temporal lobe epilepsy. Neurology. 2007; 68:122-127. [PubMed: 17210892]

Williamson, PD. Intensive monitoring of complex partial seizures: diagnosis and classification. In: Gumnit, RJ., editor. Intensive Neurodiagnostic Monitoring. New York: Raven Press; 1987. p. 69-84. 
Yu O, Mauss Y, Namer IJ, Chambron J. Existence of contralateral abnormalities revealed by texture analysis in unilateral intractable hippocampal epilepsy. Magn Res Imaging. 2001; 19:1305-1310. 


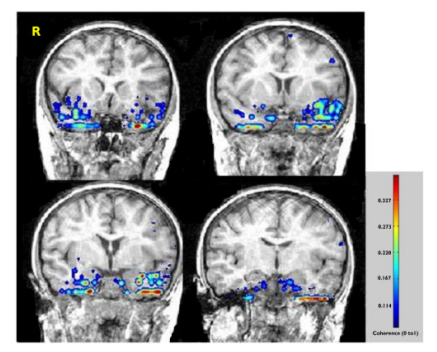

Figure 1.

Case 1. Preoperative coronal MR images overlaid with the results of coherence analysis. The study identifies an asymmetry in signal intensity (inset) weighted towards the left temporal lobe despite the absence of MTS. The patient remains seizure-free following resection of the inferopolar and mesiobasal portions of the left temporal lobe. 


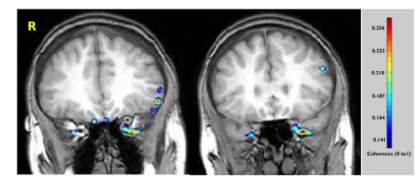

Figure 2.

Case 6. Preoperative coronal MR images overlaid with the results of coherence analysis in a case wherein the ECD method was indeterminate. The study identifies an asymmetry in signal intensity weighted toward the mesiopolar region of the left temporal lobe despite the absence of MTS. The patient remains seizure-free following resection of the inferopolar and uncal portions of the left temporal lobe. 


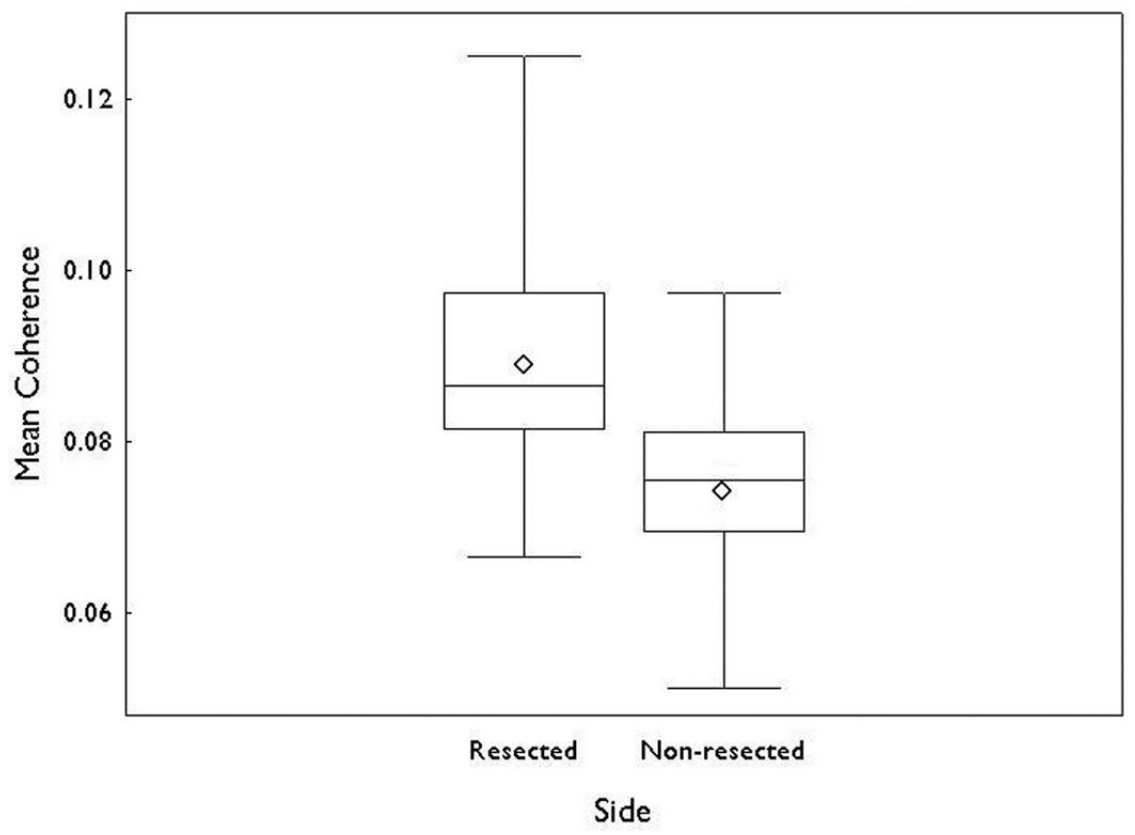

Figure 3.

Box plots showing mean $(\diamond)$, median, interquartile range and minimum and maximum values of coherence for the resected and nonresected hemispheres. The difference between the two sides was significant $(\mathrm{p}<0.001)$. 


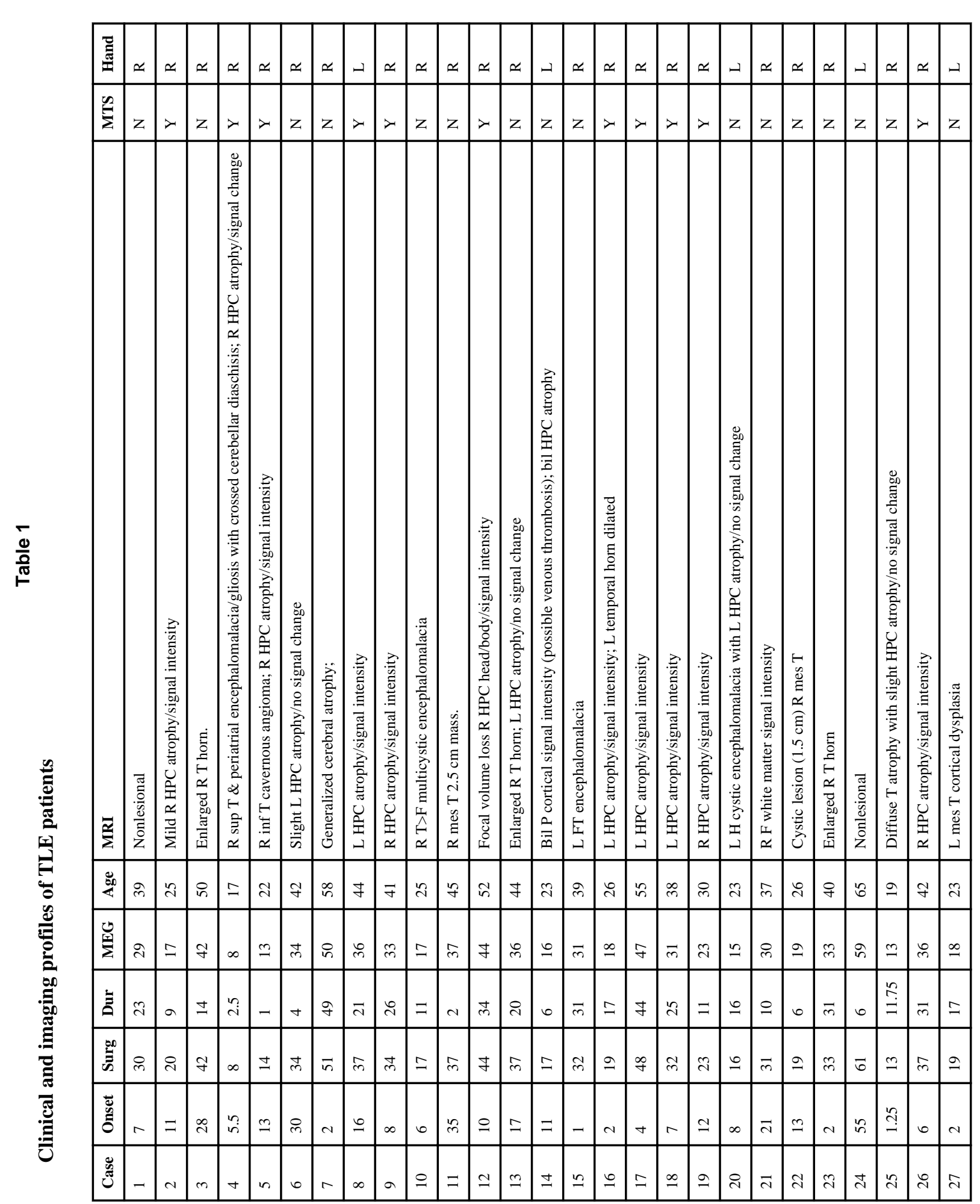




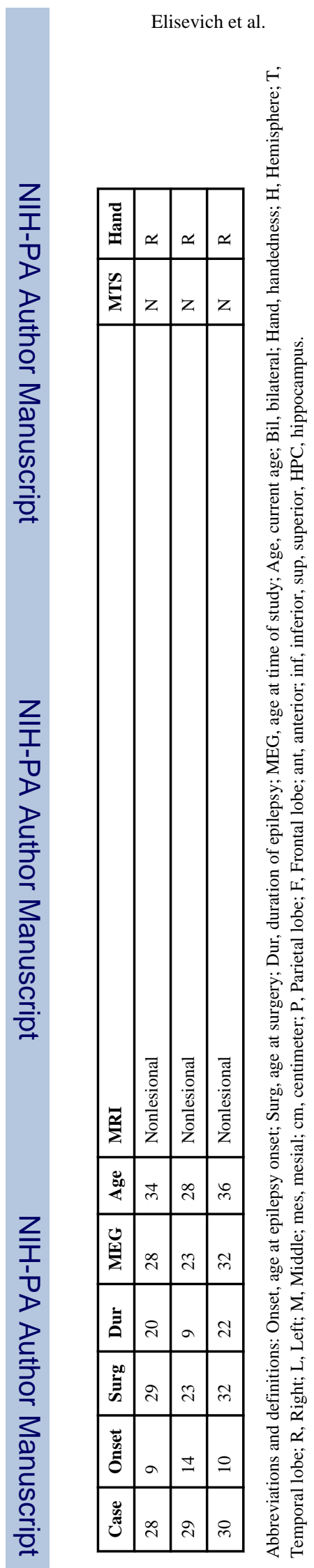

Page 18

Epilepsia. Author manuscript; available in PMC 2012 June 1. 


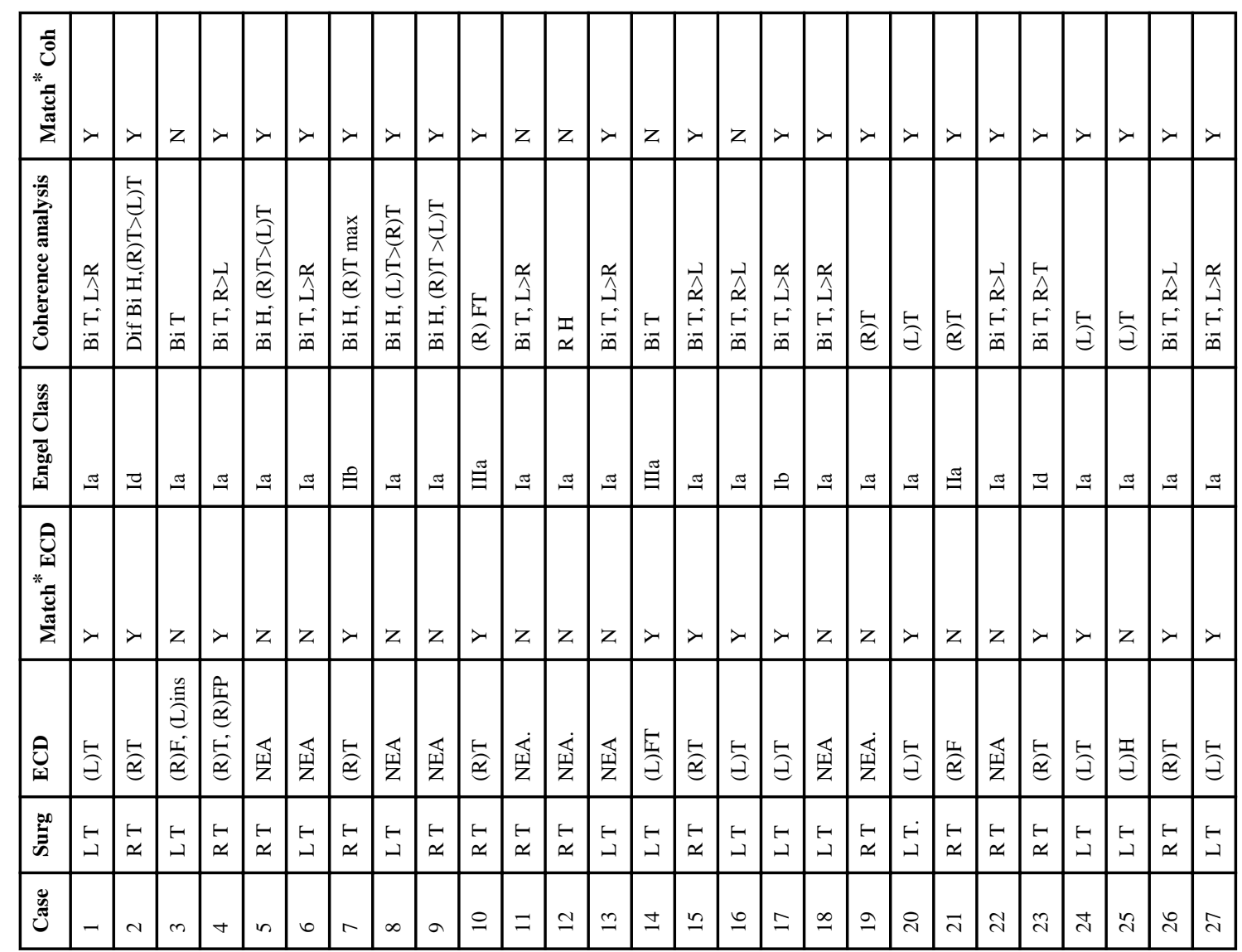




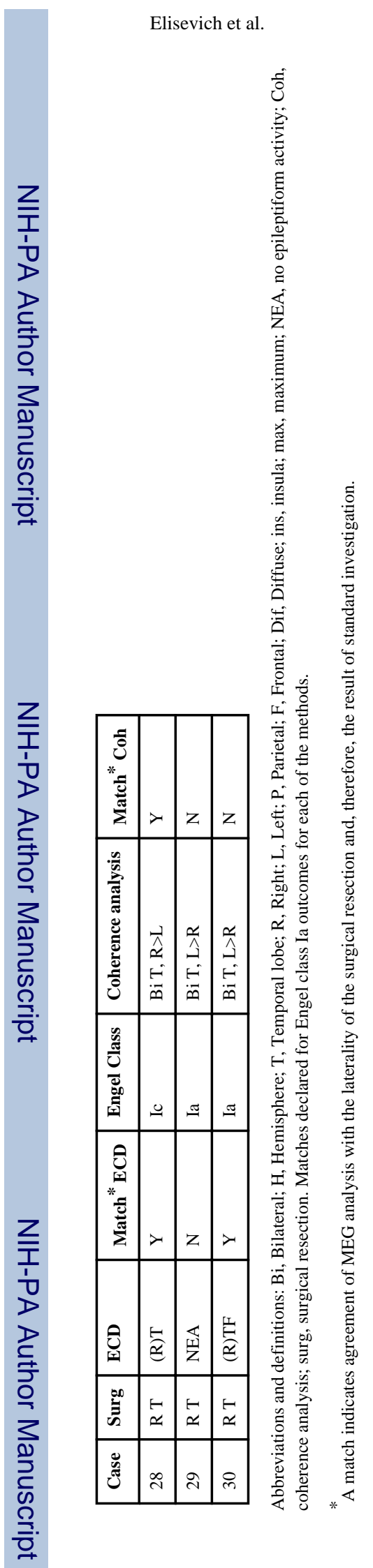

Page 20

Epilepsia. Author manuscript; available in PMC 2012 June 1. 
Table 3

Summary of Engel classes with ECD and MEG analyses

\begin{tabular}{|l|l|l|l|l|}
\hline Engel Class & \multicolumn{2}{|c|}{ EC D } & \multicolumn{2}{c|}{ Coherence analysis } \\
\hline & Match $^{*}$ & No Match & Match $^{*}$ & No Match \\
\hline Ia & 9 & 13 & 16 & 6 \\
\hline Ib & 1 & 0 & 1 & 0 \\
\hline Ic & 1 & 0 & 1 & 0 \\
\hline Id & 2 & 0 & 2 & 0 \\
\hline IIa & 0 & 1 & 1 & 0 \\
\hline IIb & 1 & 0 & 1 & 0 \\
\hline IIIa & 2 & 0 & 1 & 1 \\
\hline Total & $\mathbf{1 6}$ & $\mathbf{1 4}$ & $\mathbf{2 3}$ & $\mathbf{7}$ \\
\hline
\end{tabular}

* A match indicates agreement of MEG analysis with the laterality of the surgical resection and, therefore, the result of standard investigation. 
Table 4

Sensitivity and specificity of ECD and coherence analysis methods

\begin{tabular}{|l|r|r|r|}
\hline \multicolumn{4}{|c|}{ ECD and Engel class outcome } \\
\hline & Ia & Not Ia & Total \\
\hline Match $^{*}$ & 9 & 7 & 16 \\
\hline Not Match & 13 & 1 & 14 \\
\hline Total & 22 & 8 & 30 \\
\hline Sensitivity of Engel class Ia & 41 \\
\hline Specificity of Engel class Ia & \multicolumn{3}{|c|}{13} \\
\hline
\end{tabular}

\begin{tabular}{|l|r|r|r|}
\hline \multicolumn{4}{|c|}{ Coherence and Engel class outcome } \\
\hline & Ia & Not Ia & Total \\
\hline Match $^{*}$ & 16 & 7 & 23 \\
\hline Not Match & 6 & 1 & 7 \\
\hline Total & 22 & 8 & 30 \\
\hline Sensitivity of Engel class Ia & \multicolumn{3}{|c|}{73} \\
\hline \multicolumn{3}{|c|}{13} \\
\hline
\end{tabular}

A match indicates agreement of MEG analysis with the laterality of the surgical resection and, therefore the result of standard investigation. 